\title{
Magnetic Field - Stellar Winds Interaction
}

\author{
Asif ud-Doula \\ Penn State Worthington Scranton, Dunmore, PA 18512, USA \\ email: asif@psu.edu
}

\begin{abstract}
As per the recent study by the MiMeS collaboration, only about $10 \%$ of massive stars possess organized global magnetic fields, typically dipolar in nature. The competition between such magnetic fields and highly non-linear radiative forces that drive the stellar winds leads to a highly complex interaction. Such an interplay can lead to a number of observable phenomena, e.g. X-ray, wind confinement, rapid stellar spindown. However, due to its complexity, such an interaction cannot usually be modeled analytically, instead numerical modeling becomes a necessary tool. In this talk, I will discuss how numerical magnetohydrodynamic (MHD) simulations are employed to understand the nature of such magnetized massive star winds.
\end{abstract}

Keywords. stars: early-type, stars: magnetic fields, stars: mass loss, stars: rotation, stars: winds, outflows, X-rays: stars

\section{Introduction}

Massive hot stars are so luminous that their light can impart enough momentum to lift material off the stellar surface leading to a high mass loss. Such a mechanism is well described by the pioneering work of Castor et al. (1975, known as CAK). They have shown that scattering of photons off lines of partially ionized metals can lead to an outward force that is stronger than the inward pull of gravity.

Such outflows are expected to be steady, smooth and spherically symmetric. However, there is evidence that massive star winds have extensive structures and variability on a range of spatial and temporal scales. Relatively small-scale, stochastic structure seems most likely a natural result of the strong, intrinsic instability of the line-driving mechanism itself (Owocki 1994; Feldmeier 1995). But larger-scale structure - e.g. as evidence by explicit UV line profile variability in even low signal-to-noise IUE spectra (Kaper et al. 1996; Howarth \& Smith 1995) - seems instead likely to be the consequence of wind perturbation by processes occurring in the underlying star. For example, the photospheric spectra of many hot stars show evidence of radial and/or non-radial pulsation, and in a few cases there is evidence linking this with observed variability in UV wind lines (Telting et al. 1997; Mathias et al. 2001).

An alternate scenario is that, in at least some hot stars, surface magnetic fields could perturb, and perhaps even channel, the wind outflow, leading to rotational modulation of wind structure that is diagnosed in UV line profiles, and perhaps even to magnetically confined wind-shocks with velocities sufficient to produce the relatively hard X-ray emission seen in some hot-stars.

However, the interaction of stellar winds with magnetic fields is highly complex, and it cannot be easily modeled analytically. As such, numerical tools must be employed. In what follows, I briefly discuss how numerical computations are used to study magnetized line-driven winds. 


\section{Magneto-hydrodynamic Equations}

Massive star winds are mainly composed of fully ionized hydrogen and helium, and trace amounts of partially ionized metals $\left(\sim 10^{-4}\right)$. These winds are dense enough that the mean free paths between collisions are relatively small, and the plasma can be treated as a single fluid. The time-dependent MHD (magnetohydrodynamic) equations governing the system include the conservation of mass,

$$
\frac{\mathrm{D} \rho}{\mathrm{D} t}+\rho \nabla \cdot \mathbf{v}=0
$$

and the equation of motion

$$
\rho \frac{\mathrm{D} \mathbf{v}}{\mathrm{D} t}=-\nabla p+\frac{1}{4 \pi}(\nabla \times \mathbf{B}) \times \mathbf{B}-\frac{G M \hat{\mathbf{r}}}{r^{2}}+\mathbf{g}_{\text {external }},
$$

where $\rho, p$, and $\mathbf{v}$ are the mass density, gas pressure, and velocity of the fluid flow, and $\mathrm{D} / \mathrm{D} t=\partial / \partial t+\mathbf{v} \cdot \nabla$ is the advective time derivative. The gravitational constant $G$ and stellar mass $M$ set the radially directed $(\hat{\mathbf{r}})$ gravitational acceleration. The term $\mathbf{g}_{\text {external }}$ represents the total external force that may include the centrifugal force. In this case it is the line-force and the force due to scattering of the stellar luminosity $L$ by the free electron opacity $\kappa_{\mathrm{e}}$. The magnetic field $\mathbf{B}$ is constrained to be divergence free

$$
\nabla \cdot \mathbf{B}=0
$$

and, under the assumption of an idealized MHD flow with infinite conductivity, its inductive generation is described by

$$
\frac{\partial \mathbf{B}}{\partial t}=\nabla \times(\mathbf{v} \times \mathbf{B}) .
$$

In addition we solve an explicit equation for conservation of energy,

$$
\rho \frac{\mathrm{D}}{\mathrm{D} t}\left(\frac{e}{\rho}\right)=-p \nabla \cdot v+\Lambda_{\text {cool }} .
$$

Here the ideal gas law is used to compute the pressure, $p$, and $\Lambda_{\text {cool }}$ is radiative cooling.

\section{The Wind Magnetic Confinement Parameter}

In an interplay between magnetic field and stellar wind, the dominance of the field is determined how strong the field is relative to the wind. In order to understand the competition between these two, let us first define a characteristic parameter for the relative effectiveness of the magnetic fields in confining and/or channeling the wind outflow. Specifically, consider the ratio between the energy densities of field vs. flow as discussed by ud-Doula \& Owocki (2002),

$$
\begin{aligned}
\eta(r, \theta) & \equiv \frac{B^{2} / 8 \pi}{\rho v^{2} / 2} \approx \frac{B^{2} r^{2}}{\dot{M} v} \\
& =\left[\frac{B_{*}^{2}(\theta) R_{*}^{2}}{\dot{M} v_{\infty}}\right]\left[\frac{\left(r / R_{*}\right)^{-2 n}}{1-R_{*} / r}\right],
\end{aligned}
$$

where the latitudinal variation of the surface field has the dipole form given by $B_{*}^{2}(\theta)=$ $B_{0}^{2}\left(\cos ^{2} \theta+\sin ^{2} \theta / 4\right)$. In general, a magnetically channeled outflow will have a complex flow geometry, but for convenience, the second equality in eq. (3.1) simply characterizes the wind strength in terms of a spherically symmetric mass loss rate $\dot{M}=4 \pi r^{2} \rho v$. The third equality likewise characterizes the radial variation of outflow velocity in terms of the 
phenomenological velocity law $v(r)=v_{\infty}\left(1-R_{*} / r\right)$, with $v_{\infty}$ the wind terminal speed; this equation furthermore models the magnetic field strength decline as a power-law in radius, $B(r)=B_{*}\left(R_{*} / r\right)^{(n+1)}$, where, e.g., for a simple dipole $n=2$.

With the spatial variations of this energy ratio thus isolated within the right square bracket, we see that the left square bracket represents a dimensionless constant that characterizes the overall relative strength of field vs. wind. Evaluating this in the region of the magnetic equator $\left(\theta=90^{\circ}\right)$, where the tendency toward a radial wind outflow is in most direct competition with the tendency for a horizontal orientation of the field, one can thus define a equatorial 'wind magnetic confinement parameter',

$$
\eta_{*} \equiv \frac{B_{*}^{2}\left(90^{\circ}\right) R_{*}^{2}}{\dot{M} v_{\infty}}=0.4 \frac{B_{100}^{2} R_{12}^{2}}{\dot{M}_{-6} v_{8}}
$$

where $\dot{M}_{-6} \equiv \dot{M} /\left(10^{-6} M_{\odot} / \mathrm{yr}\right), B_{100} \equiv B_{0} /(100 \mathrm{G}), R_{12} \equiv R_{*} /\left(10^{12} \mathrm{~cm}\right)$, and $v_{8} \equiv$ $v_{\infty} /\left(10^{8} \mathrm{~cm} / \mathrm{s}\right)$. As these stellar and wind parameters are scaled to typical values for an OB supergiant, e.g. $\zeta$ Pup, the last equality in eq. (3.2) immediately suggests that for such winds, significant magnetic confinement or channeling should require fields of order $\sim 100 \mathrm{G}$. By contrast, in the case of the sun, the much weaker mass loss $\left(\dot{M}_{\odot} \sim\right.$ $\left.10^{-14} M_{\odot} / \mathrm{yr}\right)$ means that even a much weaker global field $\left(B_{0} \sim 1 \mathrm{G}\right)$ is sufficient to yield $\eta_{*} \simeq 40$, implying a substantial magnetic confinement of the solar coronal expansion. But in Bp stars the magnetic field strength can be of order $\mathrm{kG}$ with $\dot{M}_{\odot} \sim 10^{-10} M_{\odot} / \mathrm{yr}$ leading $\eta_{*} \leqslant 10^{6}$. Thus, the confinement in Bp stars is very extreme.

It should be emphasized that $\dot{M}$ used in the above formalism is predicted theoretical value obtained for a spherically symmetric non-magnetic wind.

\subsection{Alfven Radius}

What determines the extent of the effectiveness of magnetic, is the Alfvén radius, $R_{A}$, where flow and Alfvén velocities are equal. This can be derived from eq. 3.1 where the second square bracket factor shows the overall radial variation; $n$ is the power-law exponent for radial decline of the assumed stellar field, e.g. $n=2$ for a pure dipole, and $\beta$ is the velocity-law index, with typically $\beta \approx 1$. For a star with a non-zero field, we have $\eta_{*}>0$, and so given the vanishing of the flow speed at the atmospheric wind base, this energy ratio always starts as a large number near the stellar surface, $\eta\left(r \rightarrow R_{*}\right) \rightarrow \infty$. But from there outward it declines quite steeply, asymptotically as $r^{-4}$ for a dipole, crossing unity at the Alfvén radius defined implicitly by $\eta\left(R_{A}\right) \equiv 1$.

For a canonical $\beta=1$ wind velocity law, explicit solution for $R_{\mathrm{A}}$ along the magnetic equator requires finding the appropriate root of

$$
\left(\frac{R_{A}}{R_{*}}\right)^{2 n}-\left(\frac{R_{A}}{R_{*}}\right)^{2 n-1}=\eta_{*},
$$

which for integer $2 n$ is just a simple polynomial, specifically a quadratic, cubic, or quartic for $q=1,1.5$, or 2. Even for non-integer values of $2 n$, the relevant solutions can be approximated (via numerical fitting) to within a few percent by the simple general expression,

$$
\frac{R_{\mathrm{A}}}{R_{*}} \approx 1+\left(\eta_{*}+1 / 4\right)^{1 /(2 n)}-(1 / 4)^{1 /(2 n)} .
$$

For weak confinement, $\eta_{*} \ll 1$, we find $R_{\mathrm{A}} \rightarrow R_{*}$, while for strong confinement, $\eta_{*} \gg 1$, we obtain $R_{\mathrm{A}} \rightarrow \eta_{*}^{1 /(2 n)} R_{*}$. In particular, for the standard dipole case with $n=2$, we expect the strong-confinement scaling $R_{\mathrm{A}} / R_{*} \approx \eta_{*}^{1 / 4}$. 
Clearly $R_{\mathrm{A}}$ represents the radius at which the wind speed $v$ exceeds the local Alfvén speed $V_{A}$. It also characterizes the maximum radius where the magnetic field still dominates over the wind. For chemically peculiar Ap/Bp stars where stellar fields are of order $\mathrm{kG}$, the $\eta_{*} \gg 1$, e.g. for $\sigma$ Ori $\mathrm{E}$ it is about $10^{7}$, implying an Alfvèn radius $\sim 60 R_{*}$. Thus, in $\mathrm{Bp}$ (and $\mathrm{Ap}$ ) stars wind is trapped to large radii creating extensive magnetospheres.

\section{MHD Simulations}

The initial magnetohydrodynamic (MHD) simulations by ud-Doula \& Owocki (2002) assumed, for simplicity, that radiative heating and cooling would keep the wind outflow nearly isothermal at roughly the stellar effective temperature. The simulations studied the dynamical competition between field and wind by evolving MHD simulations from an initial condition when a dipole magnetic field is suddenly introduced into a previously relaxed, one-dimensional spherically symmetric wind.

Immediately after the introduction of the field, the dynamic interplay between the wind and the field leads to two distinct regions. Along the polar region, the wind streams radially outward freely, stretching the field lines into a radial configuration, as can be inferred from the left panel of illustrative Fig. 1. If the field is strong enough, around the magnetic equator a region of closed magnetic loops is formed wherein the flow from opposite hemispheres collides to make strong shocks, quite similar to what was predicted in the semi-analytic, fixed-field models of Babel \& Montmerle (1997a). The shocked material forms dense disk-like structure which is opaque to line-driving. But, its support against gravity by the magnetic tension along the convex field lines is inherently unstable, leading to a complex pattern of fall back along the loop lines down to the star, again as suggested by the left panel of Fig. 1 .

Note that even for weak field models with moderately small confinement, $\eta_{*} \leqslant 1 / 10$, the field still has a noticeable global influence on the wind, enhancing the density and decreasing the flow speed near the magnetic equator.

\section{X-ray Emission from Massive Stars}

But to model the actual X-ray emission from shocks that form from the magnetic channeling and confinement, subsequent efforts (see ud-Doula 2003; Gagné et al. 2005) have relaxed this simplification to include a detailed energy equation that follows the radiative cooling of shock-heated material. In particular, building upon the initial suggestion by Babel \& Montmerle (1997b) that such Magnetically Confined Wind Shocks (MCWS) might explain the relatively hard X-ray spectrum observed by Rosat for the O7V star $\theta^{1}$ Ori C, Gagné et al. (2005) have applied such energy-equation, MHD simulations toward a detailed, dynamical model of the more extensive Chandra X-ray observations of this star. Based on the spectropolarimetric measurement (see Donati et al. 2002) of a ca. $1100 \mathrm{G}$ field for $\theta^{1}$ Ori $\mathrm{C}$, combined with empirical and theoretical estimates of the wind momentum and stellar radius, the simulations assumed a moderately large magnetic confinement parameter, $\eta_{*} \approx 10$.

Overall, the associated X-ray emission of this MHD model matches quite well the key properties of the Chandra observations for $\theta^{1}$ Ori $\mathrm{C}$ (Gagné et al. 2005), including: the relatively hard X-ray spectrum that arises from the high post-shock temperatures $T \sim 20-30 \mathrm{MK}$; the relative lack of broadening or blue-shift from X-ray lines emitted from the nearly static, shock-heated material; and the X-ray light curve eclipse that stems from the moderate source radius $r \approx 1.5 R_{*}$ for the bulk of the X-ray emission. 

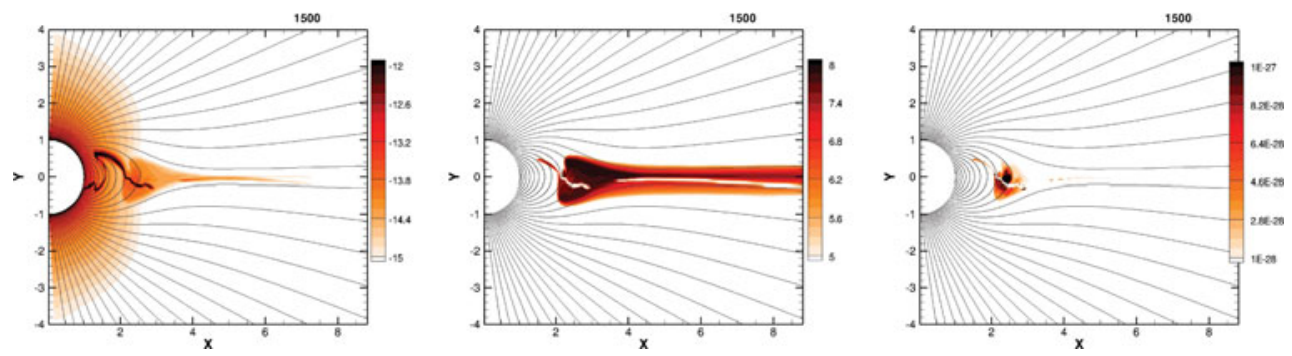

Figure 1. Color plots of log density (left) and log temperature (middle) for arbitrary snapshot of structure in the standard model with $\eta_{*}=100$. The right panel plots the proxy X-ray emission $X E M_{T_{x}}$ (weighted by the radius $r$ ), on a linear scale for a threshold X-ray temperature $T_{x}=1.5 \mathrm{MK}$.

\subsection{Shock Retreat}

In a further development ud-Doula et al. (2014) examine the effects of radiative cooling on X-ray emission from MCWS in massive stars with radiatively driven stellar winds. They show that for stars with high enough mass loss to keep the shocks radiative, the MHD simulations indicate a linear scaling of X-ray luminosity with mass-loss rate; but for lower luminosity stars with weak winds, X-ray emission is reduced and softened by a shock retreat resulting from the larger post-shock cooling length, which within the fixed length of a closed magnetic loop forces the shock back to lower pre-shock wind speeds. The basic idea behind such a shock retreat is illustrated in Fig. 2.

Through a parameter study of cooling, they show that overall scalings of time-averaged X-rays in the numerical MHD simulations are well matched by the $L_{X}$ computed from a semi-analytic "X-ray Analytic Dynamic Magnetosphere" (XADM) model. However, the values of $L_{X}$ are about a factor 5 lower in the MHD models, mostly likely reflecting an overall inefficiency of X-ray emission from the repeated episodes of dynamical infall.

\section{3D MHD}

To get a full picture of the wind-field interaction, one needs ultimately full 3D MHD simulations. Using the specific parameters chosen to represent the prototypical slowly rotating magnetic $\mathrm{O}$ star $\theta^{1}$ Ori $\mathrm{C}$, for which centrifugal and other dynamical effects of rotation are negligible ud-Doula et al. (2013) have computed the first fully 3D MHD model of its wind. The computed global structure in latitude and radius resembles that found in previous 2D simulations, with unimpeded outflow along open field lines near the magnetic poles, and a complex equatorial belt of inner wind trapping by closed loops near the stellar surface, giving way to outflow above the Alfvén radius. In contrast to this previous $2 \mathrm{D}$ work, the $3 \mathrm{D}$ simulation described here now also shows how this complex structure fragments in azimuth, forming distinct clumps of closed loop infall within the Alfvén radius, transitioning in the outer wind to radial spokes of enhanced density with characteristic azimuthal separation of $15^{\circ}-20^{\circ}$. Applying these results in a $3 \mathrm{D}$ code for line radiative transfer, they show that emission from the associated 3D 'dynamical magnetosphere' (DM) matches well the observed $\mathrm{H} \alpha$ emission seen from $\theta^{1}$ Ori $\mathrm{C}$, fitting both its dynamic spectrum over rotational phase and the observed level of cycle-to-cycle stochastic variation. Comparison with previously developed 2D models for the Balmer emission from a dynamical magnetosphere generally confirms that time averaging over $2 \mathrm{D}$ snapshots can be a good proxy for the spatial averaging over $3 \mathrm{D}$ azimuthal wind 


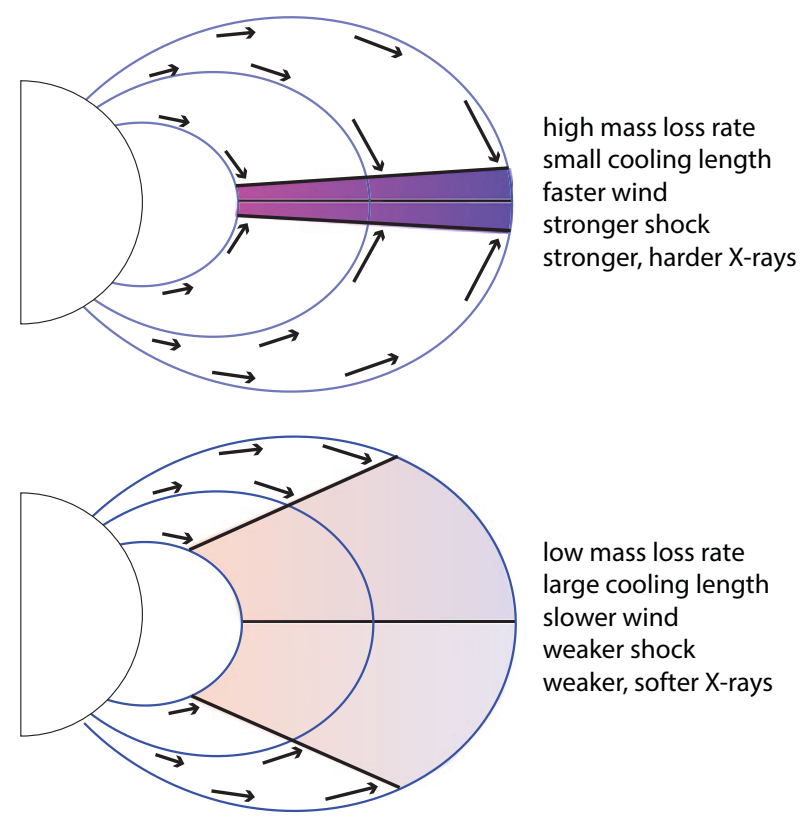

Figure 2. Schematic illustration of the "shock retreat" from inefficient cooling associated with a lower mass loss rate $\dot{M}$, showing a hemispheric, planar slice of a stellar dipole magnetic field. Wind outflow driven from opposite foot-points of closed magnetic loops is channeled into a collision near the loop top, forming magnetically confined wind shocks (MCWS). For the high $\dot{M}$ case in the upper panel, the efficient cooling keeps the shock-heated gas within a narrow cooling layer, allowing the pre-shock wind to accelerate to a high speed and so produce strong shocks with strong, relatively hard X-ray emission. For the low $\dot{M}$ case in the lower panel, the inefficient cooling forces a shock retreat down to lower radii with slower pre-shock wind, leading to weaker shocks with weaker, softer X-ray emission.

structure, as illustrated in Fig. 3. Nevertheless, fully 3D simulations will still be needed to model the emission from magnetospheres with non-dipole field components.

\section{Role of Rotation}

As noted above, MHD simulations of ud-Doula \& Owocki (2002); ud-Doula (2003) indicate that a dipole magnetic field can confine the flow within closed loops that extend out to about the Alfvèn radius, $R_{A} \approx \eta_{*}^{1 / 4} R_{*}$. In rotating models such closed loops tend also to keep the outflow in rigid-body rotation with the underlying star, and so $R_{A}$ also roughly represents the radius of maximum rotational spin-up of the wind azimuthal speed.

To further characterize such rotational effects, one can define a Keplerian corotation radius $R_{K}$ at which rigid-body rotation would yield an equatorial centrifugal acceleration that just balances the local gravitational acceleration from the underlying star,

$$
\frac{G M}{R_{K}^{2}}=\frac{v_{\phi}^{2}}{R_{K}}=\frac{V_{\mathrm{rot}}^{2} R_{K}}{R_{*}^{2}},
$$

where $V_{\text {rot }}$ is the stellar surface rotation speed at the equator. This can be solved to yield

$$
R_{K}=W^{-2 / 3} R_{*},
$$

where $W \equiv V_{\text {rot }} / V_{\text {crit }}$, with $V_{\text {crit }} \equiv \sqrt{G M / R_{*}}$ the critical rotation speed. 

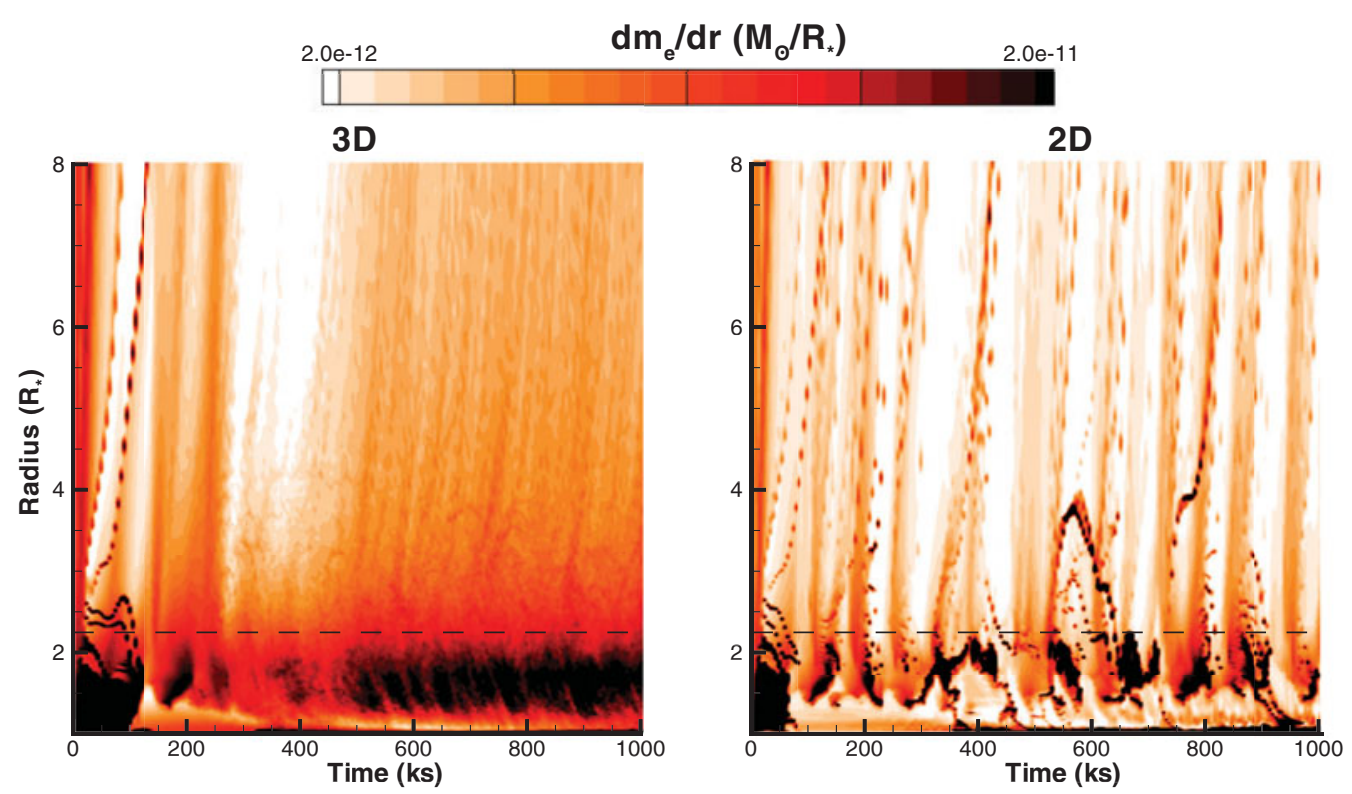

Figure 3. Equatorial mass distribution $\mathrm{d} m_{\mathrm{e}} / \mathrm{d} r$ (in units $M_{\odot} / R_{*}$ ) for the azimuthally averaged 3D model (left) and for a corresponding 2D model (right), plotted versus radius and time, with the dashed horizontal line showing the Alfvèn radius $R_{\mathrm{A}} \approx 2.23 R_{*}$. Following a similar adjustment to the initial condition, the long-term evolution of the $2 \mathrm{D}$ models is characterized by a complex pattern of repeated strings of infall, whereas the $3 \mathrm{D}$ azimuthally averaged model settles into a relatively smooth asymptotic state characterized by an enhanced mass near and below the Alfvén radius.

The Alfvén radius $R_{A}$ essentially defines the extent of the magnetosphere. If the star is a slow rotator and its $R_{K}>R_{A}$ then all the material within co-rotating region does not have enough centrifugal support to remain in orbit. As such, they rain down back onto the stellar surface leading to dynamical magnetosphere with specific implications about, for instance, $\mathrm{H} \alpha$ emission (for more details see Sundqvist et al. 2012).

On the other hand, if $R_{A}>R_{K}$ as is the case for fast rotating chemically peculiar magnetic Bp stars, the material trapped within above $R_{K}$ is forced into co-rotation leading to rigidly rotating magnetosphere. The prototypical Bp star $\sigma$ Ori E is a prime example of such a case. However, relatively low mass loss rate $\left(\dot{M} \sim 10^{-10} M_{\odot} \mathrm{yr}^{-1}\right.$ coupled with extremely strong magnetic field $\left(B \sim 10^{4} \mathrm{G}\right)$, leads to very high magnetic confinement $\left(\eta_{*} \sim 10^{7}\right)$. This in turn implies very high Alfvén speed, and thus very short Courant timestep, needed to preserve numerical stability, rendering direct MHD modelling impractical. As such, other methods must be employed to model such stars. These are discussed in the next two sections.

One other obvious impact of magnetic field in stellar wind is the angular momentum loss. The simulations done in a series of papers (see ud-Doula et al. 2006, 2008, 2009) reveal that magnetic field plays a significant role in braking and the timescale for such braking for a star with an aligned-dipole field can characterized by $\tau_{\text {brake }} \approx 0.15 \tau_{\text {mass }} \eta_{*}^{-1 / 2}$, where $\tau_{\text {mass }}$ is the mass-loss timescale (see ud-Doula et al. 2009). Nearly $80 \%$ of the angular momentum is carried away by stresses in the magnetic field, confirming that magnetic braking is an efficient process. 


\section{Rigidly Rotating Magnetosphere}

Townsend \& Owocki (2005) present a semi-analytic method for modelling the circumstellar environment of early-type stars, which is essentially a generalized extension of Babel and Montmerle model discussed above. They assume that the magnetic field lines remain rigid and corotate with the star without being influenced by the dynamics of the wind. This is essentially equivalent to the assumption of $\eta_{*} \rightarrow \infty$.

This RRM model can be applied to an arbitrary field geometry and tilt angle between the field and rotation axes. There are no dynamic forces involved in this model, instead effective gravitational + centrifugal potential is calculated based on the constrained motion of the plasma. Accumulation surfaces are assumed to lie along the location of minimum effective potential. Typically, for a tilted-dipole field case, such a surface will take the form of a geometrically thin warped disk that lies between magnetic and rotational axes.

When the accumulation process (in most cases stellar wind) is known, one can evaluate the density throughout the circumstellar environment allowing us to calculate observational diagnostics such as emission-line spectra. Indeed, Townsend and Owocki (2005) Townsend \& Owocki (2005) show that RRM model can applied to explain $\mathrm{H} \alpha$ emission from the strongly magnetic Bp star $\sigma$ Ori E remarkably well.

\section{Arbitrary Rigid-Field Hydrodynamics}

As a further improvement to the RRM model, Townsend et al. (2007) introduced a new Rigid-Field Hydrodynamics method to modelling the circumstellar environments of strongly magnetic massive stars as defined above. Just like in the RRM method, the field lines are treated as rigid, but now the flow along the lines is computed self-consistently using hydrodynamical equations including line force due to radiation. In this ansatz, flow along each field line is considered to be an independent 1D flow. They perform a large number of such 1D calculations for differing field lines, then piece them together to build up a time-dependent 3D model of a magnetosphere.

Since the flow along each field line can be solved independently of other field lines, the computational cost of this approach is a fraction of an equivalent full MHD simulation. However, this method suppresses any coupling between the flow on adjacent field lines which in practice may have some important ramifications. But overall RFHD model provides a good match with observations of Bp star magnetospheres.

This method is now superseded by Arbitrary Rigid-Field Hydrodynamics (ARFHD) method which now allows any configuration of self-consistent magnetic topology. It also improves some of the numerical algorithms and radiative cooling and includes thermal conduction (see Chris Bard's contribution in this volume).

\section{Conclusions}

Science is a lifelong journey, but unfortunately for some of us that journey gets interrupted abruptly. As a small tribute to two of our beloved colleagues and friends, Stanislav Štefl and Olivier Chesneau, who recently left us quite abruptly, I too end this report abruptly.

\section{Acknowledgements}

This work has been partially supported by NASA through Chandra Award numbers TM4-15001A issued by the Chandra X-ray Observatory Center which is operated by the Smithsonian Astrophysical Observatory for and behalf of NASA under contract 
NAS8-03060. This work was also carried out with partial support by NASA ATP Grants NNX11AC40G to the University of Delaware.

\section{References}

Babel, J. \& Montmerle, T. 1997a, ApJ (Letters) 485, 29

Babel, J. \& Montmerle, T. 1997b, A\&SA 323, 121

Castor, J. I., Abbott, D. C., \& Klein, R. I. 1975, ApJ 195, 157

Donati, J.-F., Babel, J., Harries, T. J., et al. 2002, MNRAS 333, 55

Feldmeier, A. 1995, A\&\& 299, 523

Gagné, M., Oksala, M. E., Cohen, D. H., et al. 2005, ApJ 628, 986

Howarth, I. D. \& Smith, K. C. 1995, ApJ 439, 431

Kaper, L., Henrichs, H. F., Nichols, J. S., et al. 1996, A\&AS 116, 257

Mathias, P., Aerts, C., Briquet, M., et al. 2001, A\&A 379, 905

Owocki, S. P. 1994, Ap\&SS 221, 3

Sundqvist, J. O., ud-Doula, A., Owocki, S. P., et al. 2012, MNRAS 423, L21

Telting, J. H., Aerts, C., \& Mathias, P. 1997, A\&A 322, 493

Townsend, R. H. D. \& Owocki, S. P. 2005, MNRAS 357, 251

Townsend, R. H. D., Owocki, S. P., \& ud-Doula, A. 2007, MNRAS, 382, 139

ud-Doula, A. 2003, Ph.D. thesis, University of Delaware

ud-Doula, A., Owocki, S., Townsend, R., Petit, V., \& Cohen, D. 2014, MNRAS 441, 3600

ud-Doula, A. \& Owocki, S. P. 2002, ApJ 576, 413

ud-Doula, A., Owocki, S. P., \& Townsend, R. H. D. 2008, MNRAS 385, 97

ud-Doula, A., Owocki, S. P., \& Townsend, R. H. D. 2009, MNRAS 392, 1022

ud-Doula, A., Sundqvist, J. O., Owocki, S. P., Petit, V., \& Townsend, R. H. D. 2013, MNRAS 428,2723

ud-Doula, A., Townsend, R. H. D., \& Owocki, S. P. 2006, ApJ (Letters) 640, L191

\section{Discussion}

LOBEL: Thank you for the nice presentation. In your simulations with confined magnetic fields, do you find large-scale coherent density or velocity structures that build up and stay stable in the wind beyond the Alfvén radius? I am hinting at CIRs or structures that can cause DACs in spectra of hot massive stars.

UD-DoulA: That is not something entirely obvious. In 2D simulations, by design, structures within Alfvén radius are coherent azimuthally but in 3D simulations, as shown in ud-Doula et al. (2013), such structures fragment in azimuth, forming distinct clumps of closed loop infall within the Alfvén radius, transitioning in the outer wind to radial spokes of enhanced density with characteristic azimuthal separation of $15^{\circ}-20^{\circ}$. Now, rotation will add centrifugal ejections of some of these clumps to the mix. Will they indeed form CIRs? This is something that will need further investigation.

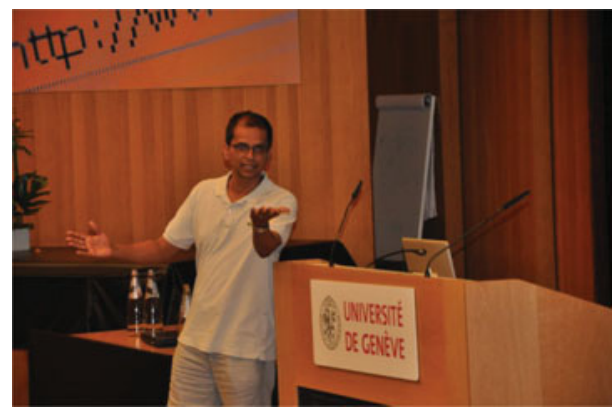

Asif ud-Doula 